\title{
Capsule Commentary on Magin et al., Changes in Australian Early-Career General Practitioners' Benzodiazepine Prescribing: a Longitudinal Analysis
}

\author{
Benjamin J. Oldfield, MD MHS \\ Yale School of Medicine, New Haven, CT, USA. \\ J Gen Intern Med 33(10):1783 \\ DOI: $10.1007 / \mathrm{s} 11606-018-4602-8$ \\ (c) Society of General Internal Medicine 2018
}

$\mathrm{T}$ his multi-site cohort study of general practitioner trainees by Magin et al. ${ }^{1}$ examined changes in benzodiazepine prescribing from 2010 through 2015. The investigators examined self-completed case reports of 1161 Australian generalpractitioner registrars (postgraduate learners in outpatient practice-based training) across five diverse training programs over time. After adjusting for registrar- and patient-level characteristics, they found that the within-registrar adjusted odds of reported benzodiazepine prescribing did not change over time, but the within-program adjusted odds of prescribing decreased by $6 \%$ per year. Assuming that these trends reflect efforts towards more judicious prescribing, the investigators conclude a reduction in benzodiazepine prescriptions by program - a welcome trend — but a lack of change by registrar suggests opportunities for targeted education.

This study addresses an important gap in our understanding of benzodiazepine prescribing patterns, and its novel focus on primary care trainees shifts our gaze "upstream" towards early-career clinicians who may be establishing durable prescribing practices. ${ }^{2}$ The study's internal validity is strengthened by the registrars' high response rate $(96 \%)$; generalizability to Australian general practitioner training programs is supported by its large sample across diverse geographic areas. However, postgraduate medical education is a heterogeneous enterprise wherein prescribing often depends on complex interactions between trainees, supervisors, and educational activities, so attributing the observed trends to policy, practice, or education efforts is speculative.

Due to increases in overdose deaths involving benzodiazepines, inappropriate prescribing of benzodiazepines has been

termed the "other prescription drug problem" in the United States, and safer prescribing is needed globally. ${ }^{3}$ This study conveys to clinicians, educators, and policymakers that there is room for improvement in postgraduate medical education regarding safe benzodiazepine prescribing. A deeper dive into the higher performing programs in this study may identify successful strategies. Furthermore, ongoing efforts to improve opioid prescribing safety such as through academic detailing ${ }^{4}$ or prescription drug monitoring programs ${ }^{5}$ - efforts supported by accumulating evidence and resources-may provide models and existing infrastructure for educational programs that promote evidence-based benzodiazepine prescribing. These programs should be evaluated and implemented.

Corresponding Author: Benjamin J. Oldfield, MD MHS; Yale School of Medicine, New Haven, CT, USA (e-mail: benjamin.oldfield@yale. edu).

\section{Compliance with Ethical Standards:}

Conflict of Interest: The author has no conflicts of interest to disclose.

\section{REFERENCES}

1. Magin P, Tapley A, Dunlop AJ, Davey A, van Driel M, Holliday E, et al. Changes in Australian early-career general practitioners' benzodiazepine prescribing: a longitudinal analysis. J Gen Intern Med. (SPI 4577).

2. Wiese HJC, Piercey RR, Clark CD. Changing Prescribing Behavior in the United States: Moving Upstream in Opioid Prescription Education. Clin Pharmacol Ther. 2018;103(6):982-9. doi:https://doi.org/10.1002/cpt. 1015

3. Lembke A, Papac J, Humphreys $\mathbf{K}$. Our Other Prescription Drug Problem. N Engl J Med. 2018;378(8):693-5. doi:https://doi.org/10. 1056/NEJMp1715050

4. Trotter Davis M, Bateman B, Avorn J. Educational Outreach to Opioid Prescribers: The Case for Academic Detailing. Pain Physician. 2017;20(2S):S147-S51.

5. Bao Y, Pan Y, Taylor A, Radakrishnan S, Luo F, Pincus HA, et al. Prescription Drug Monitoring Programs Are Associated With Sustained Reductions In Opioid Prescribing By Physicians. Health Aff (Millwood). 2016;35(6):1045-51. doi:https://doi.org/10.1377/hlthaff.2015.1673 